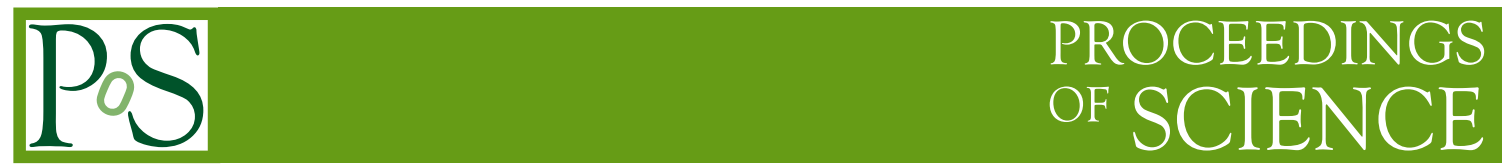

\title{
Review of production of hadron resonances in $e^{+} e^{-}$ collisions
}

\author{
Galina Pakhlova* \\ MIPT, LPI RAS \\ E-mail: galina.pakhlova@phystech.edu, gpakhlova@lebedev.ru
}

Valentina Zhukova

LPI RAS

E-mail: zhukova@lebedev.ru

\begin{abstract}
We present recent results on hadron production in $e^{+} e^{-}$annihilation including double charmonium production, $\gamma \gamma$ fusion, hadron production with initial-state radiation and from decays of $B$-mesons. We discuss the status of both standard and exotics hadron searches. Our review covers new results from Belle, BaBar, LHCb, BESIII collaborations.
\end{abstract}

XVII International Conference on Hadron Spectroscopy and Structure - Hadron2017

25-29 September, 2017

University of Salamanca, Salamanca, Spain

${ }^{*}$ Speaker. 


\section{Introduction}

For years theory has considered quarkonia as the best laboratory for testing QCD. Unlike the case of light resonances, the theory has hoped for an accurate descriptions. The last 15 years we have faced with a challenge that is best characterized by the appearance of the widely used new term -"quarkoniumlike states". The boundary between quarkonium and quarkoniumlike states is determined by the flexibility of the theory to explain the experimentally measured properties of a particular state and its decays. Upon the failure of the theory to describe the state as standard quarkonium, it is declared to be quarkoniumlike. One can hope for a reverse transition to standard quarkonium, if there are new ideas explaining the data.

Over the past decade Belle, BaBar, CLEO-c, CDF, D0, BESIII, and LHCb experiments have discovered dozens of quarkonium and quarkoniumlike states with masses above the open charm and bottom thresholds. Recent data comes mainly from BESIII experiment thanks to the large sample collected in a wide energy range in $e^{+} e^{-}$annihilation and from Belle and BaBar collaborations which use different charmonium production mechanisms such as $B$ decays, hadron production with initial-state radiation, $\gamma \gamma$ fusion, double charmonium production. New data are expected from the Super $B$-factory soon.

\section{Standard charmonium states}

To understand problems which we have in the spectrum of charmoniumlike states let us first demonstrate that there are new states which can be described by the theory as the standard charmonium. Despite apparent evidence, this claim requires proof last decade. A good recent example is the $\psi\left(1^{3} D_{2}\right)$ discovered by Belle in $B$ decays in the $\gamma \chi_{c 1}$ final state [1] and reliably confirmed by BESIII [2] in $e^{+} e^{-} \rightarrow \pi^{+} \pi^{-} \gamma \chi_{c 1}$. Its decay mode, mass, width are in good agreement with expectations, that allows to reliably identify this state as the standard charmonium.

\subsection{Vector charmonium states}

Vector charmonium states can be produced directly in $e^{+} e^{-}$annihilation. They were found about 40 years ago as structures in the total hadronic cross section above the open charm threshold. However, even today we can not conclude that $\psi$ states are well understood. In fact $\psi$ states are wide and have many common final states. Therefore their overlapping and interference substantially change the peak pattern. The BESII collaboration has attempted to take into account interference in the fit with the help of model predictions for the exclusive decays of all $\psi$ states [3]. The interference is large and makes the total contribution different from the simple sum of the Breit-Wigner resonances. The experimental data on exclusive open charm cross sections can help to avoid the model dependence. These results appeared for the first time in the works of Belle and BaBar 10 years ago $[4,5,6,7,8,9,10,11,12]$. However, only last year the first attempt was performed to describe all the measured exclusive cross sections simultaneously using the coupled channel model [13]. The measured two- and three-body final states completely saturate the inclusive hadronic cross section [14]. This allows to hope that the proposed method based on the coupled-channel model will yield a reasonable result as more accurate experimental results will be 
obtained. The main difficulty in this description was the need to account for three independent components in the $e^{+} e^{-} \rightarrow D^{*} D^{*}$ cross section, while only their sum were experimentally measured. This year, Belle presented new results on the cross sections of $e^{+} e^{-} \rightarrow D D^{*}$ and $e^{+} e^{-} \rightarrow D^{*} D^{*}$ [15]. Accuracy (both statistical and systematic) was improved by a factor of two compared to the first Belle study [5]. The new measurements are in good agreement with the previous results of both Belle [5] and BaBar [11], but the most important is angular analysis of $D^{*}$ decays, that allowed to decompose the $e^{+} e^{-} \rightarrow D^{*} D^{*}$ cross section into three independent components depending on the polarization of the two $D^{*}$. The structures for the different components are significantly different: only the $D_{T}^{*} D_{L}^{*}$ component survived at large energy as expected.

$\psi$ states are considered as standard charmonium. They decay dominantly into a pair of $D$ mesons and do not look exotic. However, Belle recently discovered unusual $\psi$ decays: $\psi(4040)$ and $\psi(4160)$ peaks in the $e^{+} e^{-} \rightarrow J / \psi \eta$ cross section with anomalous partial width of $\sim 1$ $\mathrm{MeV}$ [16]. Recently BES confirmed Belle results [17].

\section{Charmoniumlike states}

There are dozens of explicitly exotics states that cannot be explained within the framework of the quark model and the number of such states continues to grow. So far, in order to take them into account one has admit the possibility of the existence of states other than simple bound quark-antiquark pairs such as molecular states, multiquark states or hadrocharmonium.

\subsection{Vector charmoniumlike states}

The list of the vector charmoniumlike $Y$ states produced via the $e^{+} e^{-} \rightarrow Y$ process are presented in the Table 1. The existence of the $Y(4260), Y(4360)$ and $Y(4660)$ states were reliably established by Belle $[18,19]$ and BaBar $[20,21]$ via radiation return. The mass and width of the $Y(4660)$ resonance are consistent within errors with the parameters of the $X(4630)$ state observed by Belle in the $e^{+} e^{-} \rightarrow \Lambda_{c}^{+} \Lambda_{c}^{-}$[9].

Interpretation of the vector $Y$ states as standard charmonia faces a number of problems: the charmonium spectrum with $J^{P C}=1^{--}$quantum numbers has been entirely populated with the $\psi$ states and there is no room to accommodate newly observed $Y$ states; the $Y$ states with masses above open charm threshold do not decay to charm mesons unlike expectations; the partial width of the $Y \rightarrow J / \psi \pi^{+} \pi^{-}$decays ( $>1 \mathrm{MeV}$ ) exceeds by two orders of magnitude the analogous values for the standard charmonium $\psi(3770)(\psi(2 S)) \rightarrow J / \psi \pi^{+} \pi^{-}$.

Recently BESIII presented new accurate scan of $e^{+} e^{-} \rightarrow J / \psi \pi^{+} \pi^{-}$near $Y(4260)$ [22]. Already in the BaBar measurements [20] the $Y(4260)$ peak looked asymmetrical: a sharp left rise and a relatively smooth fall on the right. Finally, BESIII found an explanation of the strange lineshape as the two overlapping states, the $Y(4220)$ and $Y(4360)$, decaying to common final state, the lighter one $Y(4220)$ was found to be lighter and narrower than the $Y(4260)$. The BES results for the $e^{+} e^{-} \rightarrow \psi(2 S) \pi^{+} \pi^{-}$[23] and $e^{+} e^{-} \rightarrow h_{c} \pi^{+} \pi^{-}$[24] cross sections are no less intriguing. In both cross sections BESIII demonstrated the presence of the same $Y(4220)$ and $Y(4390)$ states as in the $J / \psi \pi^{+} \pi^{-}$case. New BESIII data indicate also the presence of the $Y(4220)$ peak in the $e^{+} e^{-} \rightarrow \chi_{c} \omega$ cross sections [25]. 


\begin{tabular}{||c|c|c|c|c||}
\hline State & $M, \mathrm{MeV}$ & $\Gamma, \mathrm{MeV}$ & Process & \\
\hline$Y(4260)$ & $4251 \pm 9$ & $120 \pm 12$ & $e^{+} e^{-} \rightarrow J / \psi \pi^{+} \pi^{-}$ & {$[20,18]$} \\
\hline$Y(4220)$ & $4222.0 \pm 3.1 \pm 1.4$ & $44.1 \pm 4.3 \pm 2.0$ & $e^{+} e^{-} \rightarrow J / \psi \pi^{+} \pi^{-}$ & {$[22]$} \\
$Y(4220)$ & $4209.5 \pm 7.4 \pm 1.4$ & $80.1 \pm 24.6 \pm 2.9$ & $e^{+} e^{-} \rightarrow \psi(2 S) \pi^{+} \pi^{-}$ & {$[23]$} \\
$Y(4220)$ & $4218.4_{4.5}^{+5.5} \pm 0.9$ & $66.0_{8.3}^{+12.3} \pm 0.4$ & $e^{+} e^{-} \rightarrow h_{c} \pi^{+} \pi^{-}$ & {$[24]$} \\
$Y(4220)$ & $4230 \pm 8 \pm 6$ & $38 \pm 12 \pm 2$ & $e^{+} e^{-} \rightarrow \chi_{c} \omega$ & {$[25]$} \\
\hline$Y(4360)$ & $4346 \pm 6$ & $102 \pm 10$ & $e^{+} e^{-} \rightarrow \psi(2 S) \pi^{+} \pi^{-}$ & {$[21,19]$} \\
\hline$Y(4360)$ & $4320.0 \pm 10.4 \pm 7.0$ & $101.4_{19.7}^{+25.3} \pm 10.2$ & $e^{+} e^{-} \rightarrow J / \psi \pi^{+} \pi^{-}$ & {$[22]$} \\
$Y(4390)$ & $4383.8 \pm 4.2 \pm 0.8$ & $84.2 \pm 12.5 \pm 2.1$ & $e^{+} e^{-} \rightarrow \psi(2 S) \pi^{+} \pi^{-}$ & {$[23]$} \\
$Y(4390)$ & $4391.5_{6.8}^{+6.3} \pm 0.9$ & $139.5_{20.6}^{+16.2} \pm 0.6$ & $e^{+} e^{-} \rightarrow h_{c} \pi^{+} \pi^{-}$ & {$[24]$} \\
\hline$Y(4660)$ & $4643 \pm 9$ & $72 \pm 11$ & $e^{+} e^{-} \rightarrow \psi(2 S) \pi^{+} \pi^{-}$ & {$[21,19]$} \\
\hline$X(4630)$ & $4634_{-11}^{+9}$ & $92_{-32}^{+41}$ & $e^{+} e^{-} \rightarrow \Lambda_{c}^{+} \Lambda_{c}^{-}$ & {$[9]$} \\
\hline
\end{tabular}

Table 1: Exotic vector states in $e^{+} e^{-}$annihilation

Another important BESIII result is the $e^{+} e^{-} \rightarrow D D^{*} \pi$ cross section [26] measured quite accurately with a small scan step. It was previously measured by Belle [7] with low accuracy. Surprisingly, in this cross section there is an indication on the presence of the $Y(4220)$. However, the interpretation of the second peak as the new $Y(4390)$ looks strange. In place of the $Y(4390)$ to be the standard $\psi(4415)$ state, which must appear in this cross section. Indeed, the $\psi(4415)$ decays into $D D_{2}$ (2460) (the Belle study of the $\left.\psi(4415) \rightarrow D D_{2}(2460) \rightarrow D D \pi[6]\right)$, which means that it should be visible not only in the $D D \pi$ final state, but also in the $D D^{*} \pi$ final state.

Thanks to BESIII, a significant revision of the $Y$ states took place. The $Y(4220)$ and $Y(4390)$ states with the improved accuracy of their masses and widths are reliably established. But in addition it was discovered that each of them has many decays in different final states in contrast to the first Belle an $\mathrm{BaBar}$ results. The last observations remove the basis of the idea of hadrocharmonium (each $Y$ resonance has its only favorite decay channel) and have to help to determine the nature of these states.

\subsection{Charged charmoniumlike states}

Another numerous family of exotic states are charged charmoniumlike states. They decay strongly into the final state with a pair of $c \bar{c}$ but have a nonzero charge. The first state in this class was the $Z^{+}(4430)$ found in $B \rightarrow \psi(2 S) \pi^{+} K$ decays in the $\psi(2 S) \pi^{+}$final state by Belle [27]. At the moment it is reliably established and well studied, its mass, width and the quantum numbers $J^{P}=$ $1^{+}$are measured quite accurately. In addition, LHCb conducted an analysis that allowed measuring the change in the complex phase as a function of the mass of the decay products $\psi(2 S) \pi^{+}$, which behaves as expected for a true resonance [28]. There are the $Z(4050)^{+}$and $Z(4250)^{+}$candidates for charged charmoniumlike states in the decays of $B$ mesons found in the $\chi_{c 1} \pi^{+}$final state [29] and the $Z(4200)^{+}$found in the $J / \psi \pi^{+}$final state [30]. They still need confirmation. Even more charged charmoniumlike states were found in $e^{+} e^{-}$annihilation. The first was the $Z(3900)^{+}$, found simultaneously by Belle [31] and by BESIII [32] in the $J / \psi \pi^{+}$final state. Today almost dozen 


\begin{tabular}{|c|c|c|c|c|}
\hline State & $M, \mathrm{MeV}$ & $\Gamma, \mathrm{MeV}$ & Process & \\
\hline$Z(3885)^{+}$ & $3883.9 \pm 4.5$ & $25 \pm 12$ & $\begin{array}{l}Y(4260) \rightarrow \pi^{-}\left(\bar{D}^{* 0} D^{+}\right) \\
Y(4260) \rightarrow \pi^{-}\left(D^{*+} \overline{D^{0}}\right)\end{array}$ & [36] \\
\hline$Z(3885)^{0}$ & $3885.7 \pm 9.8$ & $35 \pm 19$ & $e^{+} e^{-} \rightarrow\left(D \bar{D}^{*}\right)^{0}$ & [37] \\
\hline$Z(3900)^{+}$ & $3891.2 \pm 3.3$ & $40 \pm 8$ & $Y(4260) \rightarrow \pi^{-}\left(J / \psi \pi^{+}\right)$ & {$[31,32]$} \\
\hline$Z(3900)^{0}$ & $3894.8 \pm 3.5$ & $29 \pm 12$ & $Y(4260) \rightarrow \pi^{0}\left(J / \psi \pi^{0}\right)$ & [33] \\
\hline$Z(4020)^{+}$ & $4022.9 \pm 2.8$ & $7.9 \pm 3.7$ & $Y(4260,4360) \rightarrow \pi^{-}\left(h_{c} \pi^{+}\right)$ & [34] \\
\hline$Z(4020)^{0}$ & $4023.9 \pm 4.3$ & $7.9 \pm 3.7$ & $Y(4260,4360) \rightarrow \pi^{0}\left(h_{c} \pi^{0}\right)$ & [35] \\
\hline$Z(4025)^{+}$ & $4026.3 \pm 4.5$ & $24.8 \pm 9.5$ & $Y(4260) \rightarrow \pi^{-}\left(D^{* 0} D^{*+}\right)$ & [38] \\
\hline$Z(4025)^{0}$ & $4025.5 \pm 4.6$ & $23.0 \pm 6.1$ & $e^{+} e^{-} \rightarrow\left(D^{*} \bar{D}^{*}\right)^{0}$ & [39] \\
\hline$Z(4055)^{+}$ & $4032.1 \pm 2.4$ & $26.1 \pm 5.3$ & $Y(4360) \rightarrow \pi^{-}\left(\psi(2 S) \pi^{+}\right)$ & {$[40]$} \\
\hline$Z(4050)^{+}$ & $4051_{-43}^{+24}$ & $82_{-55}^{+51}$ & $\overline{B^{0}} \rightarrow K^{-}\left(\chi_{c 1} \pi^{+}\right)$ & [29] \\
\hline$Z(4200)^{+}$ & $4196_{-38}^{+35}$ & $370_{-110}^{+99}$ & $\overline{B^{0}} \rightarrow K^{-}\left(J / \psi \pi^{+}\right)$ & [30] \\
\hline$Z(4250)^{+}$ & $4248_{-45}^{+185}$ & $177_{-72}^{+321}$ & $\overline{B^{0}} \rightarrow K^{-}\left(\chi_{c 1} \pi^{+}\right)$ & [29] \\
\hline$Z(4430)^{+}$ & $4458 \pm 15$ & $166_{-38}^{+37}$ & $\bar{B}^{0} \rightarrow K^{-}\left(\psi(2 S) \pi^{+}\right)$ & {$[27,28]$} \\
\hline & & & $\bar{B}^{0} \rightarrow K^{-}\left(J / \psi \pi^{+}\right)$ & [30] \\
\hline
\end{tabular}

Table 2: Charged charmoniumlike states

states have been observed in the final states with a charmonium and $\pi$ meson or a pair of charmed mesons $[33,34,35,36,37,38,39,40]$. The current list of all observed charged charmoniumlike states is presented in the Table 2. The statistical significance of the signals is beyond doubt. At the same time, no convincing models explaining the existence of these peaks has been found yet.

\section{$3.3 \times(3872)$}

The oldest and the most interesting state in the spectrum of exotic charmonium is the $X(3872)$ state [41] which is extremely narrow. While trying to explain the broad states one can speculate about the possibility to produce peaks by dynamic effects such as cusp, re-scattering, threshold effect, etc., for the narrow $X(3872)$ such explanations are not suitable. This state is absolutely reliably established from different production mechanisms: in $B$-decays [42] and in prompt production at hadron machines $[43,44,45]$. This emphasizes the independence of the properties of this state from the initial dynamics, which is more characteristic for the real resonance than for some QCD effects. Many models attempting to explain the origin of $X(3872)$ have been proposed, tested and then excluded. So far, the only surviving model remains a molecular model, or rather a model that asserts the presence of a mixture of the $D D^{*}$ molecule to the charmonium state $\chi_{c 1}(2 P)$.

\section{$3.4 X(3915)$}

Another candidate for the exotic charmonium $Y$ (3915) was recently transferred by PDG [46] to the category of the standard charmonium. But, it seems, it has been done untimely. The $Y(3915)$ is seen in the $J / \psi \omega$ final state both in the $B \rightarrow J / \psi \omega K$ decays [47, 48] and in $\gamma \gamma$ fusion [49, 50]. BaBar has measured quantum numbers in the process of $\gamma \gamma \rightarrow Y(3915) \rightarrow J / \psi \omega$ and determined the spin of the state as $J=0$ [50]. Based on this, the PDG hastened to rewrite this state as $\chi_{c 0}(2 P)$ 
disregarding many arguments against such interpretation: the expected suppression of the production of $\chi_{c 0}(2 P)$ in $B$ decays, and the expected dominance of its decay in $D D$. In addition, later BaBar analysis was criticized: when testing the hypothesis $J=2$, not all possible production amplitudes were tested, but only those expected for real charmonium [51]. But the main argument against the interpretation of the $Y(3915)$ as $\chi_{c 0}(2 P)$ is that this place is already occupied by more likely candidate, different from the $Y(3915)$, produced and decaying as expected. In 2007 Belle found a candidate for $\chi_{c 0}(2 P)$ in the process of double charmonium production $e^{+} e^{-} \rightarrow J / \psi \chi_{c 0}(2 P) \rightarrow J / \psi D D$ [52]. Note that $\chi_{c 0}$ prefer to be produced with $J / \psi$ and decay into the $D D$ final state. While the significance of the first Belle observation was less than $5 \sigma$, the later Belle analysis using increased statistics of the full data set has confirmed the observation of $\chi_{c 0}(2 P)$ with the significance of more than $6 \sigma$ [53]. In addition, Belle showed that $J^{P C}=0^{++}$is more preferable for this candidate than $2^{++}$, although with low significance, $2.5 \sigma$.

\section{Bottomonium states}

\subsection{Standard bottomonium states}

Almost the complete bottomonium table in addition to vector states is the results of recent discoveries. Four lower vector bottomonium states $\Upsilon(1 S), \Upsilon(2 S), \Upsilon(3 S)$ and $\Upsilon(4 S)$ have been observed more then 40 years ago and perfectly fit in the expectations. The states above the open bottom threshold are more complicated both from the experimental and theoretical point of view because of the influence of open bottom thresholds. They must be taken into account in the fit of the inclusive hadron cross section.

$P$-orbital excitation of bottomonium, as in the case of charmonium, are clearly visible in the radiative decays of vector bottomonium: $\Upsilon(2 S) \rightarrow \chi_{b J}(1 P)$ and $\Upsilon(3 S) \rightarrow \chi_{b J}(2 P),(J=0,1,2)$. They were discovered by CLEO as peaks in the photon energy spectrum and all the properties of the $\chi_{b J}(1 P)$ and $\chi_{b J}(2 P)$ are well consistent with expectations [54].

The lightest state of bottomonium $\eta_{b}(1 S)$ was discovered by BaBar in the radiative decays of $\Upsilon(2 S)$ [55] and $\Upsilon(3 S)$ [56] and confirmed by CLEO [57]. The probabilities of these transitions are small and backgrounds require a very precise description. In the BaBar study the hyper-fine splitting (mass difference $m(\Upsilon(1 S))-m\left(\eta_{b}(1 S)\right)$ ) turned out to be much higher than expected (by $2 \sigma)$.

BaBar discovered the $D$-wave state of $\Upsilon\left(1^{3} D_{J}\right)$ [59] due to the large sample of the data collected at the $\Upsilon(3 S)$ energy. This state can be seen in complex cascade of radiation transitions through the $\Upsilon(3 S) \rightarrow \gamma \gamma \Upsilon\left(1^{3} D_{J}\right) \rightarrow \gamma \gamma \pi^{+} \pi^{-} \Upsilon(1 S)$ decay chain.

Quite unexpected was the observation of the $h_{b}(1 P, 2 P)$ states by Belle via $e^{+} e^{-} \rightarrow \Upsilon(5 S) \rightarrow$ $\left.h_{b}(1,2 P)\right) \pi^{+} \pi^{-}$process [60]. This discovery allowed to measure accurately the mass of $h_{b}(1 P, 2 P)$, which perfectly agrees with the center of gravity of the $\chi_{b}$ states, as expected in all models. Belle used $h_{b}(1 P, 2 P)$ as a source for studying $\eta_{b}(1 S)$ via radiative decays [58]. The measured hyperfine splitting of $\eta_{b}(1 S) \Delta M_{H F}(1 S)=57.9 \pm 2.3 \mathrm{MeV}$ removed the tension between theory and data. From radiative decays of the the $h_{b}(2 P)$ Belle has observed the $\eta_{b}(2 S)$ with a mass of $\left(9999.0 \pm 3.5_{-1.9}^{+2.8}\right) \mathrm{MeV} / \mathrm{c}^{2}[58]$. 


\subsection{Bottomoniumlike states}

In 2007 Belle reported the first observation of $e^{+} e^{-} \rightarrow \Upsilon(1 S) \pi^{+} \pi^{-}$and $\Upsilon(2 S) \pi^{+} \pi^{-}$processes near the peak of the $\Upsilon(5 S)$ resonance at $\sqrt{s} \sim 10.87 \mathrm{GeV}$ [62]. The obtained partial widths $\Gamma\left(\Upsilon(5 S) \rightarrow \Upsilon(1 S) \pi^{+} \pi^{-}\right)=0.59 \pm 0.04 \pm 0.09 \mathrm{MeV}$ and $\Gamma\left(\Upsilon(5 S) \rightarrow \Upsilon(2 S) \pi^{+} \pi^{-}\right)=0.85 \pm 0.07 \pm$ $0.16 \mathrm{MeV}$ exceeded by more than two orders of magnitude the previously measured partial widths for dipion transitions between lower $\Upsilon$ resonances, including $\Upsilon(4 S)$.

The bottomonium pattern began to resemble the case of the vector charmoniumlike $Y$ states with anomalous transitions to $\psi(1 S, 2 S) \pi^{+} \pi^{-}$final states. Since the large production rate was measured in $Y(4260) \rightarrow h_{c} \pi^{-} \pi^{-}$[61], Belle has searched for a similar transition of $\Upsilon(5 S) \rightarrow$ $h_{b}(1 P, 2 P) \pi^{+} \pi^{-}$. The discovery of this was the biggest surprise: the transitions from $\Upsilon(5 S)$ to the lowest $\Upsilon(1 S, 2 S, 3 S)$ proceed without a $b$ quarks spin flip, and into $h_{b}(1 P, 2 P)$ with a spin flip turned out to be of the same strength. The latter should be strongly suppressed but the data showed that there is no suppression. This suggested the exotic dynamics of the $\Upsilon(5 S) \rightarrow(b \bar{b}) \pi^{+} \pi^{-}$transition [63].

In fact, $e^{+} e^{-}$transitions to the $\Upsilon(1 S) \pi^{+} \pi^{-}, \Upsilon(2 S) \pi^{+} \pi^{-}, \Upsilon(3 S) \pi^{+} \pi^{-}$and to the $h_{b}(1 P) \pi^{+} \pi^{-}$, $h_{b}(2 P) \pi^{+} \pi^{-}$show two structures in the $\Upsilon(1 S, 2 S, 3 S) \pi^{+}, h_{b}(1 P, 2 P) \pi^{+}$final states corresponding to intermediate resonance states called $Z_{b}[63,64]$. There is an excellent agreement in all five modes. It should be noted that the two peaks interfere, and the complex phase between the two peaks turned out to be inverse (shifted by the number $\pi$ ) for $\Upsilon$ and $h_{b}$. This indicates the molecular nature of $Z_{b}$. The molecular hypothesis is also confirmed by the measured $Z_{b}$ quantum numbers $J^{P}=1^{+}[65]$, the discovery of the decays of $Z_{b}$ into a pair of $B B^{*}$ and $B^{*} B^{*}$ [66] and the proximity of both $Z_{b}$ to the corresponding thresholds.

However, even if the molecular model solves the problem of the existence of $Z_{b}$ states, there remains an open question: what is the nature of the $\Upsilon(5 S)$ which prefer to decay into $Z_{b}$. To search for an experimental answer to this question the cross sections of $e^{+} e^{-}$annihilation into various final states in the energy region from $\Upsilon(4 S)$ to slightly higher $\Upsilon(6 S)$ ) were investigated. The inclusive cross-section of the $e^{+} e^{-} \rightarrow b \bar{b}[67,68]$, exclusive cross section of the $e^{+} e^{-} \rightarrow$ $\Upsilon(1 S, 2 S, 3 S) \pi^{+} \pi^{-}[69]$ and of the $e^{+} e^{-} \rightarrow h_{b}(1 P, 2 P) \pi^{+} \pi^{-}$[64] were measured. The same peaks at the $\Upsilon(5 S)$ and $\Upsilon(6 S)$ energies are seen in all channels while new bottomoniumlike states are not observed. Therefore, the anomalous decays of the $\Upsilon(5 S) \rightarrow \Upsilon(1 S, 2 S, 3 S) \pi^{+} \pi^{-}$and of the $\Upsilon(5 S) \rightarrow$ $h_{b}(1 P, 2 P) \pi^{+} \pi^{-}$, which go through intermediate charged $Z_{b}$ are properties of $\Upsilon$ resonances and not of accidentally superimposed new vector bottomonium like states.

\section{Acknowledgments}

We acknowledge support from Russian Foundation for Basic Research Grant No.17-32-50019.

\section{References}

[1] V. Bhardwaj et al. [Belle Collaboration], "Evidence of a new narrow resonance decaying to $\chi_{c 1} \gamma$ in $B \rightarrow \chi_{c 1} \gamma K$," Phys. Rev. Lett. 111, no. 3, 032001 (2013) [arXiv:1304.3975 [hep-ex]].

[2] M. Ablikim et al. [BESIII Collaboration], "Observation of the $\psi\left(1^{3} D_{2}\right)$ state in $e^{+} e^{-} \rightarrow \pi^{+} \pi^{-} \gamma \chi_{c 1}$ at BESIII,” Phys. Rev. Lett. 115, no. 1, 011803 (2015) [arXiv:1503.08203 [hep-ex]]. 
[3] M. Ablikim et al. [BES Collaboration], "Determination of the $\psi(3770), \psi(4040), \psi(4160)$ and $\psi(4415)$ resonance parameters,” Phys. Lett. B 660, 315 (2008) [arXiv:0705.4500 [hep-ex]].

[4] G. Pakhlova et al. [Belle Collaboration], "Measurement of the near-threshold $e^{+} e^{-} \rightarrow D \bar{D}$ cross section using initial-state radiation,” Phys. Rev. D 77, 011103 (2008) [arXiv:0708.0082 [hep-ex]].

[5] K. Abe et al. [Belle Collaboration], "Measurement of the near-threshold $e^{+} e^{-} \rightarrow D^{(*) \pm} D^{(*) \mp}$ cross section using initial-state radiation,” Phys. Rev. Lett. 98, 092001 (2007)[hep-ex/0608018].

[6] G. Pakhlova et al. [Belle Collaboration], "Observation of $\psi(4415) \rightarrow D \bar{D}_{2}^{*}(2460)$ decay using initial-state radiation,” Phys. Rev. Lett. 100, 062001 (2008) [arXiv:0708.3313 [hep-ex]].

[7] G. Pakhlova et al. [Belle Collaboration], "Measurement of the $e^{+} e^{-} \rightarrow D^{0} D^{*-} \pi^{+}$cross section using initial-state radiation,” Phys. Rev. D 80, 091101 (2009) [arXiv:0908.0231 [hep-ex]].

[8] G. Pakhlova et al. [Belle Collaboration], "Measurement of $e^{+} e^{-} \rightarrow D_{s}^{(*)+} D_{s}^{(*)-}$ cross sections near threshold using initial-state radiation,” Phys. Rev. D 83, 011101 (2011) [arXiv:1011.4397 [hep-ex]].

[9] G. Pakhlova et al. [Belle Collaboration], "Observation of a near-threshold enhancement in the $e^{+} e^{-} \rightarrow \Lambda_{c}^{+} \Lambda_{c}$ cross section using initial-state radiation,” Phys. Rev. Lett. 101, 172001 (2008) [arXiv:0807.4458 [hep-ex]].

[10] B. Aubert et al. [BaBar Collaboration], "Study of the Exclusive Initial-State Radiation Production of the $D \bar{D}$ System," Phys. Rev. D 76, 111105 (2007) [hep-ex/0607083].

[11] B. Aubert et al. [BaBar Collaboration], "Exclusive Initial-State-Radiation Production of the $D \bar{D}$, $D^{*} \bar{D}^{*}$, and $D^{*} \bar{D}^{*}$ Systems," Phys. Rev. D 79, 092001 (2009) [arXiv:0903.1597 [hep-ex]].

[12] P. del Amo Sanchez et al. [BaBar Collaboration], "Exclusive Production of $D_{s}^{+} D_{s}^{-}, D_{s}^{*+} D_{s}^{-}$, and $D_{s}^{*+} D_{s}^{*-}$ via $e^{+} e^{-}$Annihilation with Initial-State-Radiation,” Phys. Rev. D 82, 052004 (2010) [arXiv:1008.0338 [hep-ex]].

[13] T. V. Uglov, Y. S. Kalashnikova, A. V. Nefediev, G. V. Pakhlova and P. N. Pakhlov, "Exclusive open-charm near-threshold cross sections in a coupled-channel approach," JETP Lett. 105, no. 1, 1 (2017) [arXiv:1611.07582 [hep-ph]].

[14] A. J. Bevan et al. [BaBar and Belle Collaborations], "The Physics of the B Factories," Eur. Phys. J. C 74, 3026 (2014) [arXiv:1406.6311 [hep-ex]].

[15] V. Zhukova et al. [Belle Collaboration], "Angular analysis of the $e^{+} e^{-} \rightarrow D^{(*) \pm} D^{* \mp}$ process near the open charm threshold using initial-state radiation,” Phys. Rev. D 97, 012002 (2018) [arXiv:1707.09167 [hep-ex]].

[16] X. L. Wang et al. [Belle Collaboration], "Observation of $\psi(4040)$ and $\psi(4160)$ decay into $\eta J / \psi$," Phys. Rev. D 87, no. 5, 051101 (2013) [arXiv:1210.7550 [hep-ex]].

[17] M. Ablikim et al. [BESIII Collaboration], "Measurement of the $e^{+} e^{-} \rightarrow \eta J / \psi$ cross section and search for $e^{+} e^{-} \rightarrow \pi^{0} \mathrm{~J} / \psi$ at center-of-mass energies between 3.810 and $4.600 \mathrm{GeV}$," Phys. Rev. D 91, no. 11, 112005 (2015) [arXiv:1503.06644 [hep-ex]].

[18] C. Z. Yuan et al. [Belle Collaboration], "Measurement of $e^{+} e^{-} \rightarrow \pi^{+} \pi^{-} J / \psi$ cross-section via initial state radiation at Belle," Phys. Rev. Lett. 99, 182004 (2007) [arXiv:0707.2541 [hep-ex]].

[19] X. L. Wang et al. [Belle Collaboration], "Observation of Two Resonant Structures in $e^{+} e^{-} \rightarrow \pi^{+} \pi^{-} \psi(2 S)$ via Initial State Radiation at Belle,” Phys. Rev. Lett. 99, 142002 (2007) [arXiv:0707.3699 [hep-ex]]. 
[20] B. Aubert et al. [BaBar Collaboration], "Observation of a broad structure in the $\pi^{+} \pi^{-} J / \psi$ mass spectrum around 4.26 GeV/c²," Phys. Rev. Lett. 95, 142001 (2005) [hep-ex/0506081].

[21] B. Aubert et al. [BaBar Collaboration], "Evidence of a broad structure at an invariant mass of 4.32 $\mathrm{GeV} / c^{2}$ in the reaction $e^{+} e^{-} \rightarrow \pi^{+} \pi^{-} \psi(2 S)$ measured at BaBar," Phys. Rev. Lett. 98, 212001 (2007) [hep-ex/0506081].

[22] M. Ablikim et al. [BESIII Collaboration], "Precise measurement of the $e^{+} e^{-} \rightarrow \pi^{+} \pi^{-} J / \psi$ cross section at center-of-mass energies from 3.77 to $4.60 \mathrm{GeV}$," Phys. Rev. Lett. 118, no. 9, 092001 (2017) [arXiv:1611.01317 [hep-ex]].

[23] M. Ablikim et al. [BESIII Collaboration], "Measurement of $e^{+} e^{-} \rightarrow \pi^{+} \pi^{-} \psi(3686)$ from 4.008 to $4.600 \mathrm{GeV}$ and observation of a charged structure in the $\pi^{ \pm} \psi(3686)$ mass spectrum," Phys. Rev. D 96, no. 3, 032004 (2017) [arXiv:1703.08787 [hep-ex]].

[24] M. Ablikim et al. [BESIII Collaboration], "Evidence of Two Resonant Structures in $e^{+} e^{-} \rightarrow \pi^{+} \pi^{-} h_{c}$," Phys. Rev. Lett. 118, no. 9, 092002 (2017) [arXiv:1610.07044 [hep-ex]].

[25] M. Ablikim et al. [BESIII Collaboration], "Observation of $e^{+} e^{-} \rightarrow \omega \chi_{c 1,2}$ near $\sqrt{s}=4.42$ and 4.6 GeV,” Phys. Rev. D 93, no. 1, 011102 (2016) [arXiv:1511.08564 [hep-ex]].

[26] Ke Li, [BESIII Collaboration], "New results from $Y(4260)$ decays at BESIII," Talk at XVII International Conference on Hadron Spectroscopy and Structure (Hadron 2017) Sep. 25-29, 2017, Salamanca, Spain, http://hadron2017.usal.es/

[27] S. K. Choi et al. [Belle Collaboration], "Observation of a resonance-like structure in the $\pi^{ \pm} \psi^{\prime}$ mass distribution in exclusive $B \rightarrow K \pi^{ \pm} \psi^{\prime}$ decays," Phys. Rev. Lett. 100, 142001 (2008) [arXiv:0708.1790 [hep-ex]].

[28] R. Aaij et al. [LHCb Collaboration], "Observation of the resonant character of the $Z(4430)^{-}$state," Phys. Rev. Lett. 112, no. 22, 222002 (2014) [arXiv:1404.1903 [hep-ex]].

[29] R. Mizuk et al. [Belle Collaboration], "Observation of two resonance-like structures in the $\pi^{+} \chi_{c 1}$ mass distribution in exclusive $\bar{B}^{0} \rightarrow K^{-} \pi^{+} \chi_{c 1}$ decays," Phys. Rev. D 78, 072004 (2008) [arXiv:0806.4098 [hep-ex]].

[30] K. Chilikin et al. [Belle Collaboration], "Observation of a new charged charmoniumlike state in $\bar{B}^{0} \rightarrow J / \psi K^{-} \pi^{+}$decays,” Phys. Rev. D 90, no. 11, 112009 (2014) [arXiv:1408.6457 [hep-ex]].

[31] Z. Q. Liu et al. [Belle Collaboration], "Study of $e^{+} e^{-} \rightarrow \pi^{+} \pi^{-} J / \psi$ and Observation of a Charged Charmoniumlike State at Belle,” Phys. Rev. Lett. 110, 252002 (2013) [arXiv:1304.0121 [hep-ex]].

[32] M. Ablikim et al. [BESIII Collaboration], "Observation of a Charged Charmoniumlike Structure in $e^{+} e^{-} \rightarrow \pi^{+} \pi^{-} J / \psi$ at $\sqrt{s}=4.26 \mathrm{GeV}$," Phys. Rev. Lett. 110, 252001 (2013) [arXiv:1303.5949 [hep-ex]].

[33] M. Ablikim et al. [BESIII Collaboration], "Observation of $Z_{c}(3900)^{0}$ in $e^{+} e^{-} \rightarrow \pi^{0} \pi^{0} J / \psi$," Phys. Rev. Lett. 115, no. 11, 112003 (2015) [arXiv:1506.06018 [hep-ex]].

[34] M. Ablikim et al. [BESIII Collaboration], "Observation of a Charged Charmoniumlike Structure $Z_{c}$ (4020) and Search for the $Z_{c}(3900)$ in $e^{+} e^{-} \rightarrow \pi^{+} \pi^{-} h_{c}$," Phys. Rev. Lett. 111, no. 24, 242001 (2013) [arXiv:1309.1896 [hep-ex]].

[35] M. Ablikim et al. [BESIII Collaboration], "Observation of $e^{+} e^{-} \rightarrow \pi^{0} \pi^{0} h_{c}$ and a Neutral Charmoniumlike Structure $Z_{c}(4020)^{0}$," Phys. Rev. Lett. 113, no. 21, 212002 (2014) [arXiv:1409.6577 [hep-ex]]. 
[36] M. Ablikim et al. [BESIII Collaboration], "Observation of a charged $\left(D \bar{D}^{*}\right)^{ \pm}$mass peak in $e^{+} e^{-} \rightarrow \pi D \bar{D}^{*}$ at $\sqrt{s}=4.26 \mathrm{GeV}$," Phys. Rev. Lett. 112, no. 2, 022001 (2014) [arXiv:1310.1163 [hep-ex]].

[37] M. Ablikim et al. [BESIII Collaboration], "Observation of a Neutral Structure near the $D \bar{D}^{*}$ Mass Threshold in $e^{+} e^{-} \rightarrow\left(D \bar{D}^{*}\right)^{0} \pi^{0}$ at $\sqrt{s}=4.226$ and $4.257 \mathrm{GeV}$," Phys. Rev. Lett. 115, no. 22, 222002 (2015) [arXiv:1509.05620 [hep-ex]].

[38] M. Ablikim et al. [BESIII Collaboration], "Observation of a charged charmoniumlike structure in $e^{+} e^{-} \rightarrow\left(D^{*} \bar{D}^{*}\right)^{ \pm} \pi^{\mp}$ at $\sqrt{s}=4.26 \mathrm{GeV}$," Phys. Rev. Lett. 112, no. 13, 132001 (2014) [arXiv:1308.2760 [hep-ex]].

[39] M. Ablikim et al. [BESIII Collaboration], "Observation of a neutral charmoniumlike state $Z_{c}(4025)^{0}$ in $e^{+} e^{-} \rightarrow\left(D^{*} \bar{D}^{*}\right)^{0} \pi^{0}$," Phys. Rev. Lett. 115, no. 18, 182002 (2015) [arXiv:1507.02404 [hep-ex]].

[40] X. L. Wang et al. [Belle Collaboration], "Measurement of $e^{+} e^{-} \rightarrow \pi^{+} \pi^{-} \psi(2 S)$ via Initial State Radiation at Belle,” Phys. Rev. D 91, 112007 (2015) [arXiv:1410.7641 [hep-ex]].

[41] S. K. Choi et al. [Belle Collaboration], "Observation of a narrow charmonium-like state in exclusive $B^{ \pm} \rightarrow K^{ \pm} \pi^{+} \pi^{-} J / \psi$ decays,” Phys. Rev. Lett. 91, 262001 (2003) [hep-ex/0309032].

[42] B. Aubert et al. [BaBar Collaboration], "Study of the $B \rightarrow J / \psi K^{-} \pi^{+} \pi^{-}$decay and measurement of the $B \rightarrow X(3872) K^{-}$branching fraction," Phys. Rev. D 71, 071103 (2005) [hep-ex/0406022].

[43] D. Acosta et al. [CDF Collaboration], "Observation of the narrow state $X(3872) \rightarrow J / \psi \pi^{+} \pi^{-}$in $\bar{p} p$ collisions at $\sqrt{s}=1.96$ TeV,” Phys. Rev. Lett. 93, 072001 (2004) [hep-ex/0312021].

[44] V. M. Abazov et al. [D0 Collaboration], "Observation and properties of the $X(3872)$ decaying to $J / \psi \pi^{+} \pi^{-}$in $p \bar{p}$ collisions at $\sqrt{s}=1.96$ TeV," Phys. Rev. Lett. 93, 162002 (2004) [hep-ex/0405004].

[45] R. Aaij et al. [LHCb Collaboration], "Observation of $X(3872)$ production in $p p$ collisions at $\sqrt{s}=7$ TeV,” Eur. Phys. J. C 72, 1972 (2012) [arXiv:1112.5310 [hep-ex]].

[46] C. Patrignani et al. [Particle Data Group], "Review of Particle Physics," Chin. Phys. C 40, no. 10, 100001 (2016).

[47] K. Abe et al. [Belle Collaboration], "Observation of a near-threshold $\omega J / \psi$ mass enhancement in exclusive $B \rightarrow K \omega J / \psi$ decays,” Phys. Rev. Lett. 94, 182002 (2005) [hep-ex/0408126].

[48] B. Aubert et al. [BaBar Collaboration], "Observation of $\mathrm{Y}(3940) \rightarrow J / \psi \omega$ in $B \rightarrow J / \psi \omega K$ at BABAR,” Phys. Rev. Lett. 101, 082001 (2008) [arXiv:0711.2047 [hep-ex]].

[49] S. Uehara et al. [Belle Collaboration], "Observation of a charmonium-like enhancement in the $\gamma \gamma \rightarrow \omega J / \psi$ process,” Phys. Rev. Lett. 104, 092001 (2010) [arXiv:0912.4451 [hep-ex]].

[50] J. P. Lees et al. [BaBar Collaboration], "Study of $X(3915) \rightarrow J / \psi \omega$ in two-photon collisions," Phys. Rev. D 86, 072002 (2012) [arXiv:1207.2651 [hep-ex]].

[51] Z. Y. Zhou, Z. Xiao and H. Q. Zhou, 'Could the $X(3915)$ and the $X(3930)$ Be the Same Tensor State?” Phys. Rev. Lett. 115, no. 2, 022001 (2015) [arXiv:1501.00879 [hep-ph]].

[52] P. Pakhlov et al. [Belle Collaboration], "Production of New Charmoniumlike States in $e^{+} e^{-} \rightarrow J / \psi D^{(*)} \bar{D}^{(*)}$ at $\sqrt{s} \sim 10.6 \mathrm{GeV}$," Phys. Rev. Lett. 100, 202001 (2008) [arXiv:0708.3812 [hep-ex]].

[53] K. Chilikin et al. [Belle Collaboration], "Observation of an alternative $\chi_{c 0}(2 P)$ candidate in $e^{+} e^{-} \rightarrow J / \psi D \bar{D}, ”$ Phys. Rev. D 95, 112003 (2017) [arXiv:1704.01872 [hep-ex]]. 
[54] M. Artuso et al. [CLEO Collaboration], "Photon transitions in $\Upsilon(2 S)$ and $\Upsilon(3 S)$ decays," Phys. Rev. Lett. 94, 032001 (2005) [hep-ex/0411068].

[55] B. Aubert et al. [BaBar Collaboration], "Evidence for the $\eta_{b}(1 S)$ Meson in Radiative $\Upsilon(2 S)$ Decay," Phys. Rev. Lett. 103, 161801 (2009) [arXiv:0903.1124 [hep-ex]].

[56] B. Aubert et al. [BaBar Collaboration], "Observation of the bottomonium ground state in the decay $\Upsilon(3 S) \rightarrow \gamma \eta_{b}$,” Phys. Rev. Lett. 101, 071801 (2008) Erratum: [Phys. Rev. Lett. 102, 029901 (2009)] [arXiv:0807.1086 [hep-ex]].

[57] G. Bonvicini et al. [CLEO Collaboration], "Measurement of the $\eta_{b}(1 S)$ mass and the branching fraction for $\Upsilon(3 S) \rightarrow \gamma \eta_{b}(1 S)$," Phys. Rev. D 81, 031104 (2010) [arXiv:0909.5474 [hep-ex]].

[58] R. Mizuk et al. [Belle Collaboration], "Evidence for the $\eta_{b}(2 S)$ and observation of $h_{b}(1 P) \rightarrow \eta_{b}(1 S) \gamma$ and $h_{b}(2 P) \rightarrow \eta_{b}(1 S) \gamma$,” Phys. Rev. Lett. 109, 232002 (2012) [arXiv:1205.6351 [hep-ex]].

[59] P. del Amo Sanchez et al. [BaBar Collaboration], "Observation of the $\Upsilon\left(1^{3} D_{J}\right)$ Bottomonium State through Decays to $\pi^{+} \pi^{-} \Upsilon(1 S)$, , Phys. Rev. D 82, 111102 (2010) [arXiv:1004.0175 [hep-ex]].

[60] I. Adachi et al. [Belle Collaboration], "First observation of the $P$-wave spin-singlet bottomonium states $h_{b}(1 P)$ and $h_{b}(2 P), "$ Phys. Rev. Lett. 108, 032001 (2012) [arXiv:1103.3419 [hep-ex]].

[61] T. K. Pedlar et al. [CLEO Collaboration], "Observation of the $h_{c}(1 P)$ using $e^{+} e^{-}$collisions above $D \bar{D}$ threshold,” Phys. Rev. Lett. 107, 041803 (2011) [arXiv:1104.2025 [hep-ex]].

[62] K. F. Chen et al. [Belle Collaboration], "Observation of anomalous $\Upsilon(1 S) \pi^{+} \pi^{-}$and $\Upsilon(2 S) \pi^{+} \pi^{-}$ production near the $\Upsilon(5 S)$ resonance,” Phys. Rev. Lett. 100, 112001 (2008) [arXiv:0710.2577 [hep-ex]].

[63] A. Bondar et al. [Belle Collaboration], "Observation of two charged bottomonium-like resonances in $\Upsilon(5 S)$ decays,” Phys. Rev. Lett. 108, 122001 (2012) [arXiv:1110.2251 [hep-ex]].

[64] A. Abdesselam et al. [Belle Collaboration], "Energy scan of the $e^{+} e^{-} \rightarrow h_{b}(n P) \pi^{+} \pi^{-}(n=1,2)$ cross sections and evidence for $\Upsilon(11020)$ decays into charged bottomonium-like states," Phys. Rev. Lett. 117, no. 14, 142001 (2016) [arXiv:1508.06562 [hep-ex]].

[65] A. Garmash et al. [Belle Collaboration], "Amplitude analysis of $e^{+} e^{-} \rightarrow \Upsilon(n S) \pi^{+} \pi^{-}$at $\sqrt{s}=10.865$ GeV,” Phys. Rev. D 91, no. 7, 072003 (2015) [arXiv:1403.0992 [hep-ex]].

[66] A. Garmash et al. [Belle Collaboration], "Observation of $Z_{b}(10610)$ and $Z_{b}(10650)$ Decaying to $B$ Mesons,” Phys. Rev. Lett. 116, no. 21, 212001 (2016) [arXiv:1512.07419 [hep-ex]].

[67] B. Aubert et al. [BaBar Collaboration], "Measurement of the $e^{+} e^{-} \rightarrow b \bar{b}$ cross section between $\sqrt{s}=$ 10.54 GeV and 11.20 GeV,” Phys. Rev. Lett. 102, 012001 (2009) [arXiv:0809.4120 [hep-ex]].

[68] K.-F. Chen et al. [Belle Collaboration], 'Observation of an enhancement in $e^{+} e^{-} \rightarrow \Upsilon(1 S) \pi^{+} \pi^{-}$, $\Upsilon(2 S) \pi^{+} \pi^{-}$and $\Upsilon(3 S) \pi^{+} \pi^{-}$production around $\sqrt{s}=10.89 \mathrm{GeV}$ at Belle," Phys. Rev. D 82, 091106 (2010) [arXiv:0810.3829 [hep-ex]].

[69] D. Santel et al. [Belle Collaboration], "Measurements of the $\Upsilon(10860)$ and $\Upsilon(11020)$ resonances via $\sigma\left(e^{+} e^{-} \rightarrow \Upsilon(n S) \pi^{+} \pi^{-}\right)$," Phys. Rev. D 93, no. 1, 011101 (2016) [arXiv:1501.01137 [hep-ex]]. 